\title{
Catechins as Emerging and Promising Antiparasitic Agents
}

\author{
Raúl Argüello-García*1 and Geovanna Nallely Quiñonez-Bastidas ${ }^{2}$ \\ ${ }^{11}$ Departamento de Genética y Biología Molecular, México \\ ${ }^{2}$ Departamento de Farmacia, Facultad de Química, México
}

*Corresponding author: Raul Arguello Garcia, Departamento de Genética y Biología Molecular, CINVESTAV-IPN, 07360

México City, México

\section{ARTICLE INFO}

Received: 絊 August 17, 2020

Published: 幽 September 07, 2020

Citation: Raúl Argüello-García, Geovanna Nallely Quiñonez-Bastidas. Catechins as Emerging and Promising Antiparasitic Agents. Biomed J Sci \& Tech Res 30(1)2020. BJSTR. MS.ID.004895.

\section{ABSTRACT}

Catechins are polyphenols present in green tea and other plant species as a defense against pathogen insults that profile their health-promoting and anti-infectious activities in humans. Worth noting their anti-parasite effects have emerged in recent years. In this review, we focus on the effect of catechins over growth and pathogenic mechanisms of a variety of extracellular, anaerobic (Giardia, Entamoeba) and intracellular, apicomplexan (Plasmodium) and kinetoplastid (Trypanosoma, Leishmania) protozoa and nematodes of livestock impact as Teladorsagia, Trichostrongylus, Ascaris and Haemonchus. Moreover, an inhibitory effect of (galloyl)-catechins on allergenic proteases from mites has been reported. Altogether, these data pinpoint (galloyl)-catechins as candidates for novel alternatives in therapeutic intervention and therapy-associated problems, such as secondary effects and drug resistance in parasitic infections.

\section{Introduction}

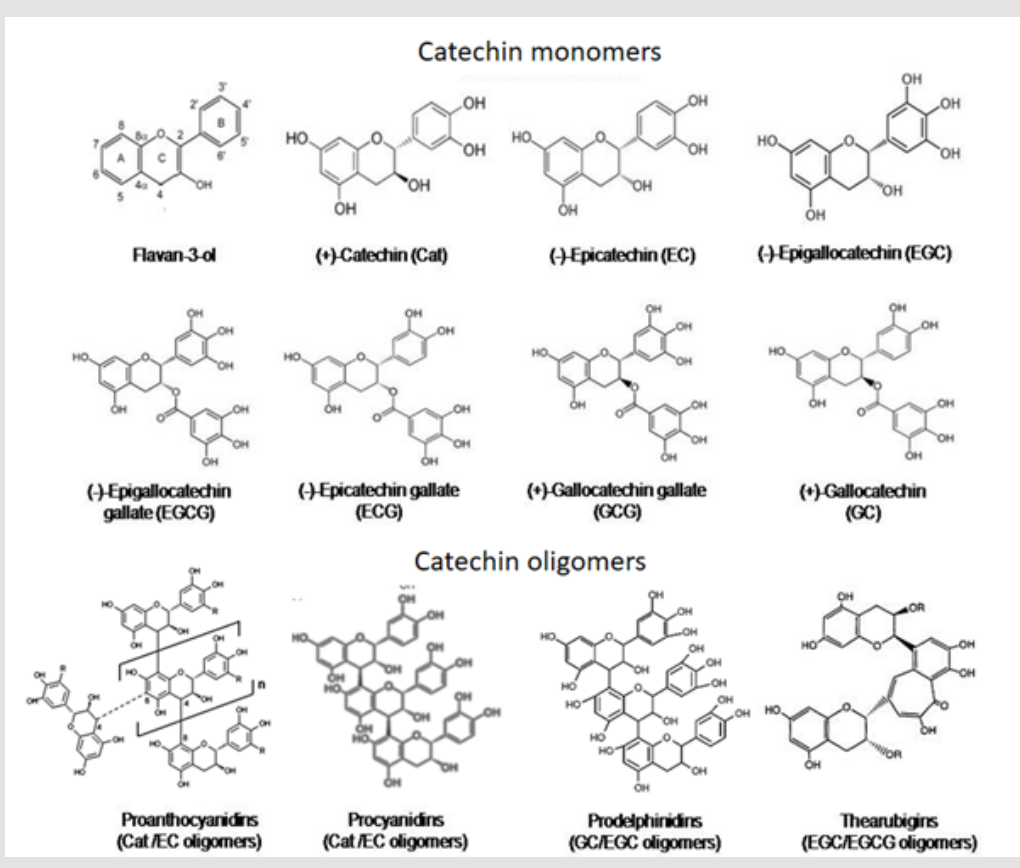

Figure 1: Chemical structures of catechin monomers and oligomers included in this work. 
Catechins (from "catechu", a boiled extract from Acacia catechu L.f) are a group of polyphenol compounds from plants classified within the subclass known as flavan-3-ols (flavanols) of the flavonoid family. Flavanols include the precursor isomers (+)-catechin (C) and (-)-epicatechin (EC), as well as, their gallate ester derivatives: (-)-epigallocatechingallate (EGCG), (-)-epicatechingallate (ECG) and (-)-epigallocatechin (EGC) along to their corresponding epimers: (+)-gallocatechingallate (GCG) and (+)-gallocatechin (GC)(Figure 1). Catechins are abundant in extracts derived from the tea plant (Camellia sinensis), in cocoa and some chocolates (from Theobroma cacao seeds), these are also found in many other plant species and are present in human diet within common foodstuffs, herbal remedies, dairy products, fruits (apple, grape, berries), vegetables and in wine as well $[1,2]$. Catechins are also found as polymeric condensation complexes. One major class of condensed catechins is the proanthocyanidin group, also called as "condensed tannins", comprising two main categories based on A-ring substitutions: phloroglucinols and resorcinols. Among these, procyanidins and prodelphinidins are the phloroglucinol-type proanthocyanidins most found in (Figure 1). Otherwise during fermentation of black tea the reddish thearubigins that result from condensation of EGC and EGCG, namely theaflavins, are formed [3] (Figure 1).

Since the past two decades, there has been an increasing interest to consider catechins as an alternative for pharmacological intervention in a spectrum of therapeutic issues as diabetes, neuropathic pain, obesity, neurodegenerative disorders and antimicrobial activities [4-8]. In general, the diverse activities displayed by catechins and condensed tannins are dependent on the catechin type, suggesting a clear influence of the galloyl moiety [9-12]. On the other hand, pathogenic organisms as endoparasites (protozoa and helminthes) and ecto-parasites (e.g. mites) are major but often neglected infectious agents causing severe health problems of high morbidity and/or mortality in clinical and veterinary practice. These are transmitted by multiple routes: waterborne, foodborne, by soil or vectors and have complex life cycles involving, importantly, multiple morphological stages that have limited the establishment of effective vaccines. Also, side effects of and resistance to prescribed drugs are reportedly growing issues that make necessary a continuous search for new and safer antiparasitic compounds [13]. In this context, the recent completion of parasite genome sequencing projects provides a useful platform towards drug target deconvolution along to molecular databases (chemicals, proteins) to decipher the mode of action of parasiticidal agents.

\section{Antiprotozoal Action of Catechins}

Protozoa include unicellular eukaryotes that account for $30 \%$ of all known parasite species (21 out of 69 ) causing human disease that usually requires therapeutic intervention [14]. In this scenario, various monomeric catechins have been tested against extracellular, intestinal, and microaerophilic protozoa as
Giardia duodenalis and Entamoeba histolytica that have pathogenic (trophozoite) and infectious (cyst) stages and are commonly associated to infectious diarrhea. In studies on inhibition of trophozoite growth in vitro using a set of 18 plant flavonoids that included three catechins, (-)-EC and (-)-EGC were the two most potent compounds whilst (+)-Cat had the sixth efficacy against these two Helianthemum glomeratum pathogens [15]. Likewise $(-)$-EC and (+)-Cat were isolated from both Geranium mexicanum roots and aerial parts of Rubus coriifolius Focke, with the former being the most effective antiamoebic and antigiardial component and the latter displaying moderate activity $[16,17]$. Other Mexican medicinal plant, Helianthemum glomeratum contained both (-)-EGC and (-)-EGCG of which the former was equipotent to (-)-EC and the latter to (+)-Cat against Giardia and Entamoeba [18]. These results give evidence on the importance of the presence of 3-0H group (as minus isomer hence in cis-configuration with B-ring) for higher antiamoebic and antigiardial activities of catechin monomers. In addition the precursor ( + )-Cat and the derivative catechin-3-0- $\alpha-\mathrm{L}-$ rhamnopyranoside isolated from the dioecious hemiparasite shrub Osyris alba displayed a toxicity almost 20 -fold lower (i.e. higher $\mathrm{IC}_{50}$ values) against cancer (Hep-2) and non-malignant (Vero) cells than against Giardia and Entamoeba.

Metronidazole, a current drug of choice against these parasites but with emerging evidence of drug-resistance [19] and variable drug susceptibility [20] exhibited a toxicity index better by far (above 200-fold); however catechins have still a promisingly favorable profile for further preclinical tests [21]. Consistent to this notion, (-)-EC had a better efficacy (i.e. lower $\mathrm{ED}_{50}$ values) in CD-1 mice experimentally infected with $G$. duodenalis than metronidazole and other flavonoids as kaempferol and tiliroside isolated from the Mexican medicinal plants G. mexicanum, Cuphea pinetorum, H. glomeratum and R. coriifolius [22]. The mechanism of catechin-induced cell death in these two protozoa harbouring mitosomes instead typical mitochondria has not been elucidated but Entamoeba trophozoites exposed to (-)-EC displayed chromatin clumping, increased glycogen deposits, reduced number and size of cytoplasmic vacuoles and alteration of cytoskeletal functions $[23,24]$. These features resemble programmed cell death (PCD)like induced by protein translation inhibitors and nitrosative stress-inducing agents in this organism.

Regarding oligomeric catechins, in roots of the plant Geranium niveum two uncommon A-type proanthocyanidins, named as

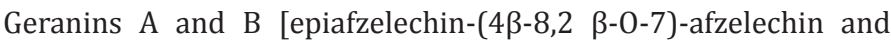

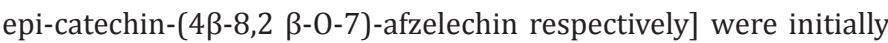
isolated and showed inhibitory effect on the growth of both parasites albeit Giardia was highly susceptible to both compounds and Geranin A had a much lower efficacy on E. histolytica trophozoites [25]. Further isolation of Geranins $C$ and D [epi-afzelechin$(4 \beta-8,2 \beta-0-7)$-gallocatechin and epi-afzelechin-(4 $\beta-8,2 \beta-0-7)$ afzelechin-(4 $\beta-8,2 \beta-0-7)$-afzelechin respectively] rendered a moderate activity for the Geranin $\mathrm{D}$ against both parasites while 
Geranin C was moderately active only against E. histolytica [26]. Thus, the efficacy order for Geranins was $\mathrm{A}>\mathrm{B}>\mathrm{D}>\mathrm{C}$ for Giardia and $\mathrm{B}>\mathrm{D}>\mathrm{C}>\mathrm{A}$ for Entamoeba.

Other group of protozoa is the kinetoplastids causing Chagas disease ( $T$. cruzi) and the cutaneous/mucocutaneous/visceral leishmaniasis complex (e.g. Leishmania major, L. amazonensis and L. donovani respectively). In Chagas disease and Leishmaniasis, these organisms have the intracellular amastigote stage within macrophages or tissue cells and extracellular trypomastigote/ epimastigote (T. cruzi) or promastigote (Leishmania, of which the Trypanosomatid group exclusively clusters parasitic organisms causing important human diseases as sleeping sickness (e.g. Typanosoma brucei) stages. Noteworthy the characteristic kinetoplast is a DNA-rich region within a single large mitochondrion that differs both structurally and functionally from mammalian ones hence renders it an attractive chemotherapeutic target [27]. In this sense the green tea's most abundant catechin, EGCG, decreased viability of intracellular Leishmania without significant induction of nitric oxide production by infected macrophages [28] and caused structural and functional collapse of mitochondria in L. amazonensis promastigotes associated to reduced parasite burden in infected mice $[29,30]$. Also, ROS-mediated oxidative stress caused by EGCG was proposed as parasiticidal mechanism in L. braziliensis promastigotes and amastigotes [31]. Recent studies denoted the importance of the galloyl group for activity against L. infantum promastigotes because the efficacy of derivatives was EGCG>ECG>Cat [32]. In Trypanosoma cruzi, from a set of 8 (galloyl)-catechins from $C$. sinensis, GCG and EGCG were the most potent compounds with $\mathrm{IC}_{50}$ values in the picomolar range against bloodstream trypomastigotes and in the micromolar range against amastigotes infecting Vero cells [33]. Further, intraperitoneal administration of EGCG in a murine model of acute Chagas disease increased survival from 11 to $60 \%$ and diminished parasitemia by 50\%; interestingly this compound induced PCD-like in epimastigotes as assessed by DNA oligosomal fragmentation [34].

Within the protozoal phylum apicomplexa (i.e. alveolate intracellular organisms that use a cytoskeleton-rich apical complex to penetrate host cells), Plasmodium is a prominent genus infecting a broad range of vertebrates including reptiles, birds, and mammals. In human beings $P$. falciparum is the deadliest species as it causes malaria and presents several stages within mosquito vector and at liver and red cells in vertebrate hosts. Other important aplicomplexan genus is Babesia that infects livestock worldwide, penetrates erythrocytes and is transmitted by ticks. In southern multimammate rodents (Mastomys coucha) infected with P. berghei, treatment with $(+)$-Cat reduced parasitemia and erythrocytic oxidative stress while improving host survival [35]. Remarkably EGCG displays effects in P. falciparum at several levels: in a structure-based drug screening using the crystal structure of the human intercellular adhesion molecule (ICAM-1), EGCG was shown to block the binding of P. falciparum-infected erythrocytes to ICAM1 that is exposed on endothelial cell surface, an event associated to pathology in malaria [36]. Moreover, this inhibition varied amongst parasite isolates in the range $37-80 \%$ at $50 \mu \mathrm{M}$ EGCG, which was suggested to mimic the L43 loop of ICAM-1 [37]. Also, the membrane permeabilizer digitonin synergized the cytotoxicity of EGCG against liver sporozoites and additively inhibited parasite motility [38]. Furthermore, EGCG exhibits disaggregating effect on amyloid-like fibrils formed by the vaccine candidate merozoite surface protein-2 (MSP-2) causing oligomer release [39]. In addition, EGCG was shown able to interact with the chaperone HSP70 of $P$. falciparum interfering eith its ATPase and chaperone functions [40]. In the case of bovine B. bovis and murine B. microti, EGCG displays an adverse effect on parasite growth in vitro $\left(\mathrm{IC}_{50}\right.$ in the micromolar range) and at $5-10 \mathrm{mg} / \mathrm{kg}$ it cleared parasites in infected mice [41].

\section{Anthelminthic Activity of Catechins}

Parasitic helminthes include the phyla Nematoda (roundworms) and Platyhelminthes (flatworms) [42]. Soil-transmitted helminthes are the most common infectious agents of humans in developing countries, mainly at tropical and subtropical areas as sub-Saharan Africa, America, chine, and oriental Asia region [43]. Helminthiasis is a global public health problem. Approximately, 15 million of people are infected by soil-transmitted helminthes, which represent a quarter of world's population [44]. The infections occur when the humans ingest infecting eggs (Ascaris lumbricoides and Trichuris trichiura) or larvae (Ancylostoma duodenale) in contaminated food, or through penetration of the skin by infective hookworm larvae in soil (Necator americanus and Ancylostoma duodenale) [45] King) affecting over 1 billion persons worldwide. Their prevalence is closely linked to the presence of severe poverty and its associated sub-standard housing and sanitation. The last decade has seen a remarkable increase in our understanding of the true disease burden of helminth infections, and there has been increasing momentum on the part of national and non-governmental developmental organizations for prevention and control of these diseases. The expansion in mass treatment programmes for their control has yielded some significant successes. However, challenges remain in terms of ecological heterogeneity in transmission, incomplete drug uptake, and the likelihood of emerging drug resistance. The development of new, more-sensitive diagnostics is now broadening our knowledge of infection prevalence and of the risk of reinfection and has enhanced our knowledge of the prevalence of concurrent helminth infections. Adoption of these new diagnostic techniques for large-scale screening and surveillance will require adaptation of current mass treatment guidelines for control as programmes move from initial morbidity control objectives toward coordinated interventions aimed at local elimination.",'author": [\{“dropping-particle":"',',family":"King",'given":"Charles H',"nondropping-particle":"',"parse-names":false,"suffix":"'”],"containertitle":"Advances in parasitology","id":"ITEM-1",'issued":\{“date-par 
ts":[[“2019”]]\},"language":"eng","page":"11-30","publisher-place":" England","title":"Helminthiasis Epidemiology and Control: Scoring Successes and Meeting the Remaining Challenges.","type":"articlejournal","volume":"103"\},"uris":["http://www.mendeley.com/ documents /?uuid=b034daec-8568-4046-addc-1003762f34cc" ]\}],"mendeley":\{“formattedCitation":"[42]","manualFormatting" :"King, 2019. Current clinical trials efforts mainly focus in mass drug administration (MDA) strategies as the best way for control of soil-transmitted helminthes (STH), addressing the school-age child sector and adults with high risk-infection occupations [46]. However, despite to the wide options of anti-helminthic drugs available (albendazole, mebendazole, benzimidazole, Praziquantel, oxamniquine, ivermectin and others), helminthiasis continue to grow worldwide and, in a similar form, drug-resistance nematodes increase with particular emphasis on livestock [45].

In this regard, this review analyzes the catechins as potential anthelminthic compounds. Evidence indicates that Cat has effects on motility and mortality of helminthes, prompting catechichs as promising natural antiparasitic agents. In line with this, investigations support the EC properties against the trematode Paramphistomum cervi [47]. Moreover, EC was related to anticestode activity against Raillietina echinobothrida, suggesting a mechanism of action associated to decreased nitric oxide synthase (NOS) and the subsequent nitric oxide (NO) production [48]. Besides, in vitro studies support that compounds CG, ECG, GC, EGC, and EGCG, isolated from Acacia nilotica fruits, have nematocidal activity against Onchocerca ochengi and the model Caenorhabditis elegans, remarking the anthelminthic actions of catechins [49]. On the other hand, the activity and migration of helminthes larvae, as Ascaris suum, is inhibited by action of GC, EGC and EGCG compounds $[50,51]$. In a similar form, polyphenols including catechins, have an important role in a very early stage of host invasion parasitic, which explains the inhibitory effects on gasto-intestinal infection of some nematodes [52]. Moreover, the inhibitory effects of CG, EGC, GCG and EGCG were observed on egg hatching and larvae development (L3) of Trichostrongylus colubriformis [53]. In a short summary, there are significant evidence about of potential use of catechins against experimental helminthiasis, notwithstanding, clinical trials that support the anti-helminthic effect of catechins have not been developed yet.

\section{Catechins and Mites}

Mites are small arthropods that along to ticks are contained in the arachnid taxon Acari; some of these are parasites, causing diseases such as scabies and skin allergies in humans and plant pathologies. In plants as Quercus ilex that form erinea (hyperplasic leaf trichomes), these structures are enriched in (-)-Cat and proanthocyanidin B3 for protection upon attack by the mite Aceria ilicis [54]. By other side, Dermatophagoides farinae is present inhouse dust and produces a serine protease (Df-protease) that induces allergy through the kallikrein-kinin system thereby inducing vascular permeability, inflammation hence allergy in human skin. Catechins inhibited Df-protease-induced kinin release in human plasma in the order EGCG>ECG>EGC>EC and EGCG effectively suppressed vascular permeability in guinea pigs, reinforcing the importance of the galloyl moiety for interaction with this enzyme [55]. In a clinical sense, patients with persistent allergic rhinitis induced by dust mite displayed alleviated symptoms upon treatment with apple polyphenols containing 52\% procyanidins and $9 \%$ catechins [56].

\section{Catechin Targets in Parasites}

Catechins may interact with different kinds of protein molecules or their biological partners that protozoa, helminthes and mites possess; here, the advent and use of newest molecular and bioinformatics tools is encouraging this field. In protozoa as Plasmodium, (+)-EGCG can interact with the DE loop of ICAM1 thereby blocking the cytoadherence of Plasmodium-infected erythrocytes to vascular endothelium [36]. Also, surface plasmon resonance assays show that (+)-EGCG inhibits ATPase and chaperone activities of two heat shock proteins at cytosol of $P$. falciparum (HSP70-1 and HSP70-z) [40]. Other studies have shown that catechins target and inhibit enzymes of energy metabolism such as recombinant rat arginase- 1 and its L. amazonensis homolog (ARG-L) but these are more actives against the parasite enzyme. Interestingly the galloyl moiety adversely affected the interactions with ARG-L thus their effectiveness was $(+)$-Cat $>(-)$-EC $>(-)$-EGCG [57] and the inverse relation was obtained by in vitro promastigote viability and in silico molecular docking with the redox enzyme trypanothione synthase [32]. For T.brucei that relies on fatty acid synthesis for proliferation and virulence, the first enzyme of this pathway, acetyl CoA carboxylase, is phosphorylated then inhibited by activation of AMP-dependent protein kinase which is directly activated by (-)-EGCG in bloodstream and procyclic forms [58].

In helminthes and mites the identification of catechins targets is still limited. Helminthes are gifted with proteins of redox metabolism that have been found targeted by condensed tannins. A nematode-specific, mitochondrial thioredoxin reductase (TrxR) from $H$. contortus is inhibited by theaflavins in a qualitatively similar mode to other known TrxR inhibitors as curcumin and auranofin among others [59]. Likewise, recombinant glutathione S-transferase (GST) from Ascaris and Onchocerca are inhibited by extracts containing proanthocyanidins from Nigerian traditional plants used against gastrointestinal helminthes [60]. The unique mite target identified so far is the Df-protease that was shown inhibited by EGCG over other less galloylated catechins [55].

Figure 2 depicts the structural alignment of representative targets of catechins, particularly EGCG, in protozoa $(P$. falciparum erythrocyte membrane protein 1), helminthes $(H$. contortus thioredoxin reductase) and mites (Df-protease) with their respective closest homologs (internalin $\mathrm{K}$ from Listeria 
monocytogenes, $82.8 \%$ structure homology; mouse mitocondrial TrxR, 92.5\% structure homology; human hepsin, 80.4\% structure homology). In general, the parasite molecules display substantial structure differences involving moieties with potential docking or targeting by catechins even when compared to host proteins, in a similar way than that reported for other known catechin targets as are leishmanial arginases and trypanothion synthase. Taken together, these analyses unveil a broad perspective for rational drug design and testing of catechins against parasite molecules that could in turn serve as platform for future therapeutic interventions in the diseases these pathogens cause.
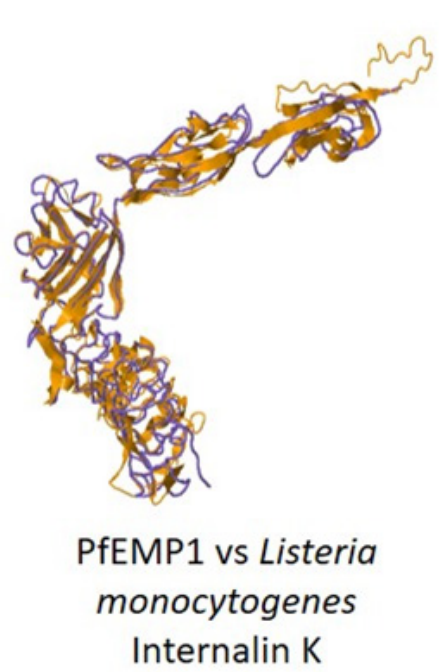

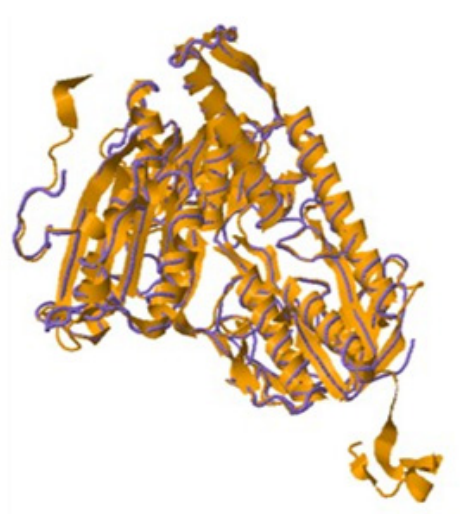

HcmitTxR vs Mus musculus mitochondrial thioredoxin reductase, C-terminal 3- $\Delta$

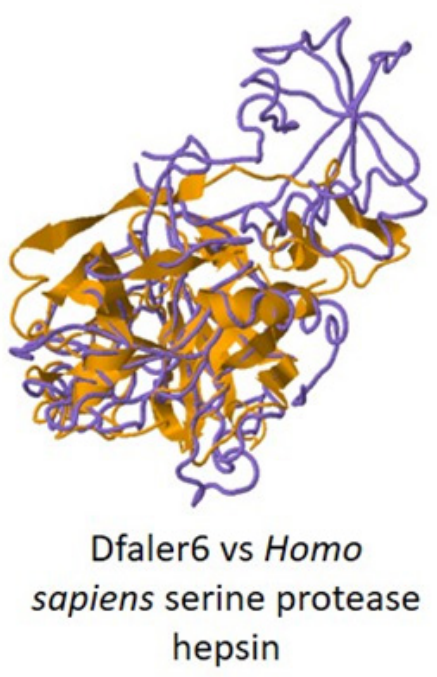

hepsin

Figure 2: Protein structure alignment of representative catechin targets in protozoa (PfEMP1), helminthes (HcmitTrxR) and mites (Dfaler6) (colored in orange) with their closest crystal homologs (Internalin K PDB ID: 4I3aA; MmTrxR PDB ID: 3dgzA; HsHepsin PDB ID: 1z8gA) (colored in purple).

\section{Conclusion}

Catechins have activity against pathogens from the plants these are synthesized and towards infectious agents affecting humans and companion and livestock animals as well. Their antiparasitic activities have been studied at deeper detail from the past two decades. Monomeric catechins, particularly EGCG, exhibit a broad range of antiprotozoal activity targeting important enzymes of redox and energy metabolism with a favored selectivity in relation with host analogs. Structure-activity relationships mostly show an increased effect by the galloyl moieties of these flavan-3ols. Condensed tannins, i.e. oligomeric catechins, have cytotoxic effects not only for protozoal but also for helminth pathogens, causing impaired motility, larval ex sheath ment and migration of ruminant and bovine nematodes. In mites, the search for allergen counteraction with catechins is a scarcely explored but very promising issue. In the current absence of proven vaccines against parasites, recent tools as ligand- and target-structure based strategies coupled to formulations with new adjuvants and vehicles will help the future development and exploitation of these important flavonoids in the control of parasitic diseases.

\section{Conflicts of Interest}

Authors declare that there are not conflicts of interest concerning this work.

\section{References}

1. Shahidi F (2000) Antioxidants in food and food antioxidants. NahrungFood 44(3): 158-163.

2. Shimizu M, Shirakami Y, Moriwaki H (2008) Targeting receptor tyrosine kinases for chemoprevention by green tea catechin, EGCG. International Journal of Molecular Sciences 9(6): 1034-1049.

3. Robertson A, Bendall DS (1993) Production and HPLC analysis of black tea theaflavins and thearubigins during in vitro oxidation. Phytochemistry 22(4): 883-887.

4. Al-Gayyar M, Matragoon S, Pillai B, Ali T, Abdelsaid M, et al. (2011) Epicatechin blocks pro-nerve growth factor (proNGF)-mediated retinal neurodegeneration via inhibition of p75 neurotrophin receptor proNGF expression in a rat model of diabetes. Diabetologia 54(3): 669-680.

5. Ramirez-Sanchez I, Maya L, Ceballos G, Villarreal F (2011) (-)-Epicatechin induces calcium and translocation independent eNOS activation in arterial endothelial cells. American Journal of Physiology-Cell Physiology 300(4): C880-c887.

6. Si W, Gong J, Tsao R, Kalab M, Yang R, et al. (2006) Bioassay-guided purification and identification of antimicrobial components in Chinese green tea extract. Journal of Chromatography A 1125(2): 204-210.

7. Quinonez-Bastidas GN, Pineda-Farias JB, Flores-Murrieta FJ, Rodriguez-Silverio J, Reyes-Garcia JG, et al. (2018) Antinociceptive effect of (-)-epicatechin in inflammatory and neuropathic pain in rats. Behavioural Pharmacology 29(2 and 3): 270-279.

8. Quiñonez-Bastidas GN, Cervantes-Durán C, Rocha-González HI, Murbartián J, Granados-Soto V (2013) Analysis of the mechanisms underlying the antinociceptive effect of epicatechin in diabetic rats. Life Sciences 93(17): 637-645. 
9. Yoshioka H, Sugiura K, Kawahara R, Fujita T, Making M, et al. (1991) Formation of Radicals and Chemiluminescence during the Autoxidation of Tea Catechins. Agricultural and Biological Chemistry 55(11): 27172723.

10. Rice-evans CA, Miller NJ, Bolwell PG, Bramley PM, Pridham JB (1995) The Relative Antioxidant Activities of Plant-Derived Polyphenolic Flavonoids. Free Radical Research 22(4): 375-383.

11. Zaveri NT (2006) Green tea and its polyphenolic catechins: Medicinal uses in cancer and noncancer applications. Life Sciences 78(18): 20732080.

12. Intra J, Kuo S-M (2007) Physiological levels of tea catechins increase cellular lipid antioxidant activity of vitamin $\mathrm{C}$ and vitamin $\mathrm{E}$ in human intestinal caco-2 cells. Chemico-Biological Interactions 169(2): 91-99.

13. Argüello-García R, Leitsch D, Skinner-Adams T, Ortega-Pierres MG (2020) Chapter Six - Drug resistance in Giardia: Mechanisms and alternative treatments for Giardiasis. In: Ortega-Pierres MGBT-A in $\mathrm{P}$ editor. Giardia Giardiasis, Part B 107: 201-282.

14. Kuris AM (2012) The global burden of human parasites: who and where are they? How are they transmitted? Journal of Parasitology 98(6):10561064 .

15. Calzada F, Meckes M, Cedillo R, Tapia-Contreras A, Mata R (1998) Screening of Mexican Medicinal Plants for Antiprotozoal Activity. Pharmaceutical Biology 36: 305-309.

16. Alanis AD, Calzada F, Cedillo-Rivera R, Meckes M (2003) Antiprotozoal activity of the constituents of Rubus coriifolius. Phytotheraphy Research 17(6): 681-682.

17. Calzada F, Cervantes-Martínez JA, Yépez-Mulia L (2005) In vitro antiprotozoal activity from the roots of Geranium mexicanum and its constituents on Entamoeba histolytica and Giardia lamblia. Journal of Ethnopharmacology 98(1-2): 191-193.

18. Meckes M, Calzada F, Tapia-Contreras A, Cedillo-Rivera R (1999) Antiprotozoal properties of Helianthemum glomeratum. Phytotherapy Research 13(2): 102-105.

19. Ali V, Nozaki T (2007) Current therapeutics, their problems, and sulfurcontaining-amino-acid metabolism as a novel target against infections by "amitochondriate" protozoan parasites. Clinical Microbiology Reviews 20(1): 164-187.

20. Argüello-García R, Cruz-Soto M, Romero-Montoya L, Ortega-Pierres G (2004) Variability and variation in drug susceptibility among Giardia duodenalis isolates and clones exposed to 5-nitroimidazoles and benzimidazoles in vitro. Journal of Antimicrobial Chemotherapy 54(4): $711-721$

21. Al-Jaber HI, Mosleh IM, Mallouh A, Abu Salim OM, Abu Zarga MH (2010) Chemical constituents of Osyris alba and their antiparasitic activities. Journal of Asian Natural Products Research 12(9): 814-820.

22. Barbosa E, Calzada F, Campos R (2007) In vivo antigiardial activity of three flavonoids isolated of some medicinal plants used in Mexican traditional medicine for the treatment of diarrhea. Journal of Ethnopharmacology 109(3): 552-554

23. Soto J, Gomez C, Calzada F, Ramirez ME (2010) Ultrastructural changes on Entamoeba histolytica HM1-IMSS caused by the flavan-3-ol, (-)-epicatechin. Planta Medica 76(6): 611-612.

24. Bolaños V, Díaz-Martínez A, Soto J, Rodríguez MA, López-Camarillo C et al. (2014) The flavonoid (-)-epicatechin affects cytoskeleton proteins and functions in Entamoeba histolytica. Journal of Proteomics 111: 74 85.

25. Calzada F, Cerda-García-Rojas CM, Meckes M, Cedillo-Rivera R, Bye R, et al. (1999) Geranins A and B, new antiprotozoal A-type proanthocyanidins from Geranium niveum. Journal of Natatural Products 62(5): 705-709.

26. Calzada F, Cedillo-Rivera R, Bye R, Mata R (2001) Geranins C and D, additional new antiprotozoal A-type proanthocyanidins from Geranium niveum. Planta Medica 67(7): 677-680.
27. Sen N, Majumder HK (2008) Mitochondrion of protozoan parasite emerges as potent therapeutic target: exciting drugs are on the horizon. Current Pharmaceutical Design 14(9): 839-846.

28. Kolodziej H, Radtke OA, Kiderlen AF (2008) 9 Stimulus (polyphenol, IFNgamma, LPS)-dependent nitric oxide production and antileishmanial effects in RAW 264.7 macrophages. Phytochemistry 69: 3103-3110.

29. Inacio JDF, Canto-Cavalheiro MM, Menna-Barreto RFS, Almeida-Amaral EE (2012) Mitochondrial damage contribute to epigallocatechin3 -gallate induced death in Leishmania amazonensis. Experimental Parasitollgy 132(2): 151-155.

30. Inacio JDF, Canto-Cavalheiro MM, Almeida-Amaral EE (2013) In vitro and in vivo effects of (-)-epigallocatechin 3-0-gallate on Leishmania amazonensis. Journal of Natural Products 76(10): 1993-1996.

31. Inacio JDF, Gervazoni L, Canto-Cavalheiro MM, Almeida-Amaral EE (2014) The effect of (-)-epigallocatechin 3-0--gallate in vitro and in vivo in Leishmania braziliensis: involvement of reactive oxygen species as a mechanism of action. PLoS Neglected Tropical Diseases 8(8): e3093.

32. Khademvatan S, Eskandari K, Hazrati-Tappeh K, Rahim F, Foroutan $\mathrm{M}$, et al (2019) In silico and in vitro comparative activity of green tea components against Leishmania infantum. Journal of Global Antimicrobial Resistence 18: 187-194.

33. Paveto C, Güida MC, Esteva MI, Martino V, Coussio J, et al (2004) AntiTrypanosoma cruzi activity of green tea (Camellia sinensis) catechins. Antimicrobial Agents and Chemotherapy 48(1): 69-74.

34. Güida MC, Esteva MI, Camino A, Flawiá MM, Torres HN, et al. (2007) Trypanosoma cruzi: in vitro and in vivo antiproliferative effects of epigallocatechin gallate (EGCg). Experimental Parasitology 117(2): 188194.

35. Srivastava PJ, Chandra S, Arif AJ, Singh C, Panday V (1999) Metal chelators/antioxidants: approaches to protect erythrocytic oxidative stress injury during Plasmodium berghei infection in Mastomys coucha. Pharmacological Research 40(3): 239-241.

36. Dormeyer M, Adams Y, Kramer B, Chakravorty S, Tse MT, et al. (2006) Rational design of anticytoadherence inhibitors for Plasmodium falciparum based on the crystal structure of human intercellular adhesion molecule 1. Antimicrobial Agents and Chemotherapy 50(2): 724-730.

37. Patil PR, Gemma S, Campiani G, Craig AG (2011) Broad inhibition of plasmodium falciparum cytoadherence by $(+)$-epigallocatechin gallate. Malaria Journal 10: 348.

38. Hellmann JK, Münter S, Wink M, Frischknecht F (2010) Synergistic and additive effects of epigallocatechin gallate and digitonin on Plasmodium sporozoite survival and motility. PLoS One 5: e8682.

39. Chandrashekaran IR, Adda CG, Macraild CA, Anders RF, Norton RS (2011) EGCG disaggregates amyloid-like fibrils formed by Plasmodium falciparum merozoite surface protein 2 . Archives of Biochemistry Biophysics 513(2): 153-157.

40.Zininga T, Ramatsui L, Makhado PB, Makumire S, Achilinou I, et al. (2017) (-)-Epigallocatechin-3-Gallate Inhibits the Chaperone Activity of Plasmodium falciparum Hsp70 Chaperones and Abrogates Their Association with Functional Partners. Molecules 22(12): 2139.

41. Aboulaila M, Yokoyama N, Igarashi I (2010) Inhibitory effects of (-)-epigallocatechin-3-gallate from green tea on the growth of Babesia parasites. Parasitology 137(5): 785-791.

42. King CH (2019) Helminthiasis Epidemiology and Control: Scoring Successes and Meeting the Remaining Challenges. Advances in Parasitollgy 103: 11-30.

43. World Health Organization (2020) Soil-Transmitted Helminth Infect.

44. Jourdan PM, Lamberton PHL, Fenwick A, Addiss DG (2018) Soiltransmitted helminth infections. Lancet (London, England) 391(10117): 252-265. 
45. Peter Hotez, Donald Bundy, Kathleen Beegle, Simon Brooker, Lesley Drake, et al. (2006) Soil-transmitted Helminth Infections and Schistosomiasis. In: Dean T Jamison, Joel G Breman, Anthony R Measham, George Alleyne, et al. (Eds) $2^{\text {nd }}(E d n)$, Disease Control Priorities in Developing Countries. Washington (DC): The International Bank for Reconstruction and Development / The World Bank, New York, USA.

46. Ásbjörnsdóttir KH, Ajjampur SSR, Anderson RM, Bailey R, Gardiner I, et al. (2018) Assessing the feasibility of interrupting the transmission of soil-transmitted helminths through mass drug administration: The DeWorm3 cluster randomized trial protocol. PLoS Neglected Tropical Diseases 12(1): e0006166-e0006166.

47. Zahir AA, Rahuman AA, Bagavan A, Geetha K, Kamaraj C, et al. (2012) Evaluation of medicinal plant extracts and isolated compound epicatechin from Ricinus communis against Paramphistomum cervi. Parasitology Research 111: 1629-1635.

48. Chetia M, Das R (2018) Effect of (-)-epicatechin, a flavonoid on the NO and NOS activity of Raillietina echinobothrida. Acta Tropica 178: 311317.

49. Dikti Vildina J, Kalmobe J, Djafsia B, Schmidt TJ, Liebau E, et al. (2017) Anti-Onchocerca and Anti-Caenorhabditis Activity of a Hydro-Alcoholic Extract from the Fruits of Acacia nilotica and Some Proanthocyanidin Derivatives. Molecules 22(5): 748.

50. Williams AR, Fryganas C, Ramsay A, Mueller-Harvey I, Thamsborg SM (2014) Direct anthelmintic effects of condensed tannins from diverse plant sources against Ascaris suum. PLoS One 9(5): e97053-e97053.

51. Ramsay A, Williams AR, Thamsborg SM, Mueller-Harvey I (2016) Galloylated proanthocyanidins from shea (Vitellaria paradoxa) meal have potent anthelmintic activity against Ascaris suum. Phytochemistry 122: 146-153.

52. Azaizeh H, Halahleh F, Abbas N, Markovics A, Muklada H, et al. (2013) Polyphenols from Pistacia lentiscus and Phillyrea latifolia impair the exsheathment of gastro-intestinal nematode larvae. Veterinaty Parasitology 191(1-2): 44-50.

\section{ISSN: 2574-1241}

DOI: 10.26717/BJSTR.2020.30.004895

Raúl Argüello-García. Biomed J Sci \& Tech Res

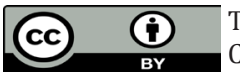

This work is licensed under Creative Commons Attribution 4.0 License

Submission Link: https://biomedres.us/submit-manuscript.php
53. Molan AL, Meagher LP, Spencer PA, Sivakumaran S (2003) Effect of flavan-3-ols on in vitro egg hatching, larval development and viability of infective larvae of Trichostrongylus colubriformis. International Journal of Parasitology 33(14): 1691-1698.

54. Karioti A, Tooulakou G, Bilia AR, Psaras GK, Karabourniotis G, et al. (2011) Erinea formation on Quercus ilex leaves: anatomical, physiological and chemical responses of leaf trichomes against mite attack. Phytochemistry 72(2-3): 230-237.

55. Noguchi Y, Fukuda K, Matsushima A, Haishi D, Hiroto M, et al. (1999) Inhibition of Df-protease associated with allergic diseases by polyphenol. Journal of Agricultural and Food Chemistry 47: 2969-2972.

56. Enomoto T, Nagasako-Akazome Y, Kanda T, Ikeda M, Dake Y (2006) Clinical effects of apple polyphenols on persistent allergic rhinitis: A randomized double-blind placebo-controlled parallel arm study. Journal of Investigation Allergology and Clinical Immunology 16(5): 283-289.

57. Dos Reis MBG, Manjolin LC, Maquiaveli C do C, Santos-Filho OA, da Silva ER (2013) Inhibition of Leishmania (Leishmania) amazonensis and rat arginases by green tea EGCG, (+)-catechin and (-)-epicatechin: a comparative structural analysis of enzyme-inhibitor interactions. PLoS One 8: e78387.

58. Vigueira PA, Ray SS, Martin BA, Ligon MM, Paul KS (2012) Effects of the green tea catechin (-)-epigallocatechin gallate on Trypanosoma brucei. International Journal of Parasitology Drugs and Drug Resistance 2: 225229.

59. Hudson AL, Sotirchos IM, Davey MW (2010) Substrate specificity of the mitochondrial thioredoxin reductase of the parasitic nematode Haemonchus contortus. Parasitology Research 107(2): 487-493.

60. Fakae BB, Campbell AM, Barrett J, Scott IM, Teesdale-Spittle PH, et al (2000) Inhibition of glutathione S-transferases (GSTs) from parasitic nematodes by extracts from traditional Nigerian medicinal plants. Phytotherapy Research 14(8): 630-634.

$\begin{array}{ll}\text { BIOMEDICAL } & \text { Assets of Publishing with us } \\ \text { RESEARCHES } & \text { - Global archiving of articles } \\ \text { - Immediate, unrestricted online access }\end{array}$

